\title{
Frequency and risk factors for major complications after stereotactic radiofrequency ablation of liver tumors in 1235 ablation sessions: a 15-year experience
}

\author{
Peter Schullian ${ }^{1} \cdot$ Edward Johnston ${ }^{2} \cdot$ Gregor Laimer $^{1}$ • Daniel Putzer ${ }^{1} \cdot$ Gernot Eberle $^{1} \cdot$ Arno Amann $^{3}$. \\ Maria Effenberger ${ }^{4} \cdot$ Manuel Maglione $^{5} \cdot$ Martin C. Freund $^{6} \cdot$ Alexander Loizides $^{6} \cdot$ Reto Bale $^{1}$ (i)
}

Received: 18 May 2020 / Revised: 27 August 2020 / Accepted: 12 October 2020 / Published online: 30 October 2020

(C) The Author(s) 2020

\begin{abstract}
Objectives To assess the frequency of major complications after multi-probe stereotactic radiofrequency ablation (SRFA) in a large cohort of patients over 15 years and to elucidate risk factors for adverse events.

Materials and methods A retrospective study was carried out between July 2003 and December 2018. Seven hundred ninety-three consecutive patients (median 65.0 years (0.3-88), 241 women and 552 men, were treated in 1235 SRFA sessions for 2475 primary and metastatic liver tumors with a median tumor size of $3.0 \mathrm{~cm}(0.5-18 \mathrm{~cm})$. The frequency of major complications was evaluated according to SIR guidelines and putative predictors of adverse events analyzed using simple and multivariable logistic regression. Results Thirty-day mortality after SRFA was $0.5 \%$ (6/1235) with an overall major complication rate of 7.4\% (91/1235). The major complication rate decreased from $11.5 \%$ (36/314) (before January 2011) to 6.0\% (55/921) $(p=0.001) .50 .5 \%(46 / 91)$ of major complications were successfully treated in the same anesthetic session by angiographic coiling for hemorrhage and chest tube insertion for pneumothorax. History of bile duct surgery/intervention, number of coaxial needles, and location of tumors in segment IVa or VIII were independent prognostic factors for major complications following multivariable logistic regression analysis. Simple logistic regression revealed the number of tumors, tumor size, location close to the diaphragm, tumor conglomerate, and segment VII as other significant predictors.

Conclusion SRFA of liver tumors is safe and can extend the treatment spectrum of conventional RFA. Adaptations over time combined with increasing experience resulted in a significant decrease in complications.

Key Points

- In 1235 ablation sessions in 793 patients over 15 years, we found a mortality rate of 0.5\% (6/1235) and an overall major complication rate of $7.4 \%$, which fell from 11.5 (36/314) to 6.0\% (55/921, p = 0.001) after January 2011, likely due to procedural adaptations.

- History of bile duct surgery/intervention $(p=0.013$, OR $=3.290)$, number of coaxial needles $(p=0.026$, OR $=1.052)$, and location of tumors in segment IVa $(p=0.016, O R=1.989)$ or VIII $(p=0.038, O R=1.635)$ were found to be independent prognostic factors.

- Simple logistic regression revealed that number of tumors, tumor size, location close to the diaphragm, tumor conglomerates, and segment VII were other significant predictors of major complications.
\end{abstract}

Reto Bale

reto.bale@i-med.ac.at

1 Section of Interventional Oncology - Microinvasive Therapy (SIP), Department of Radiology, Medical University of Innsbruck, Anichstr. 35, 6020 Innsbruck, Austria

2 The Royal Marsden Hospital, 203 Fulham Road, Chelsea, London SW3 6JJ, UK

3 Department of Internal Medicine V, Medical University of Innsbruck, Anichstr. 35, 6020 Innsbruck, Austria
4 Department of Internal Medicine I, Medical University of Innsbruck, Anichstr. 35, 6020 Innsbruck, Austria

5 Department of Visceral, Transplant and Thoracic Surgery, Center of Operative Medicine, Medical University of Innsbruck, Anichstr. 35, 6020 Innsbruck, Austria

6 Department of Radiology, Medical University of Innsbruck, Anichstr. 35, 6020 Innsbruck, Austria 
Keywords Radiofrequency ablation $\cdot$ Liver $\cdot$ Neoplasm $\cdot$ Therapy

\author{
Abbreviations \\ HCC Hepatocellular carcinoma \\ HR Hepatic resection \\ ICC Intrahepatic cholangiocarcinoma \\ ICU Intensive care unit \\ RF Radiofrequency \\ SRFA Stereotactic radiofrequency ablation \\ US Ultrasound
}

\section{Introduction}

Hepatic resection remains a major strategy in the management of both primary and metastatic liver tumors, although it is associated with morbidity rates of 14 55\% [1-3]. More recently, percutaneous radiofrequency ablation (RFA) has become widely accepted as a less invasive treatment strategy in the management of small unresectable liver tumors including hepatocellular carcinoma (HCC) and liver metastases from primaries including colorectal, breast, and neuroendocrine [4-6]. RFA has shown comparable overall survival after hepatic resection in patients with very-early-stage HCC [7] and demonstrates lower morbidity varying from 0.9 to $10.0 \%[4,8,9]$. However, the technique necessitates a safety margin whereby the ablation zone completely surrounds the tumor by $10 \mathrm{~mm}$ to achieve local tumor control and favorable clinical outcome [10-12]. Achieving adequate treatment margins may be challenging using US or CT guidance alone, particularly for large and irregularly shaped tumors or in situations when the target tumor is either difficult to visualize, awkward to access, or adjacent to vulnerable structures. Moreover, challenging locations pose a higher risk of complications where stereotactic guidance is not used $[13,14]$. Stereotaxy is already widely used in radiotherapy and neurosurgery and facilitates the execution of pre-defined three-dimensional plans to enable more complex and difficult ablations. For example, tumors high in the hepatic dome or caudate lobe require steep and often long routes, where three-dimensional planning and precision targeting allow transthoracic routes to be avoided with optimal distribution of RF probes around the target tumor.

The purpose of the current study is to assess the frequency of major complications after multi-probe SRFA with intraoperative image fusion, to evaluate adaptations over time and determine risk factors accounting for adverse events.

\section{Materials and methods}

\section{Patient cohort and inclusion criteria}

This retrospective, single-center study was approved by the Institutional Review Board. Written informed consent for SFRA was obtained from the entire study population. All cases were reviewed, and treatment plans approved by consensus in multidisciplinary tumor board meetings consisting of hepatologists, oncologists, transplant surgeons, and interventional radiologists. Therapy decisions were made considering tumor and patient-specific characteristics such as size, number, anatomical location, and performance status. Owing to our stereotactic multi-probe approach, a potentially local curative treatment could be offered without limitations on tumor size or number. Thus, BCLC-B and BCLC-C patients were offered SRFA as first-line therapy despite $\mathrm{BCLC}$ guidelines recommending treatment with transarterial chemoembolization or medical therapy. Between July 2003 and December 2018, we performed 1527 consecutive SRFA sessions for primary and secondary liver tumors. Ablations for benign liver lesions $(n=28)$, those with palliative intention to treat due to extensive tumor spread $(n=75)$, liver packing prior to SRFA $(n=56)$, and ablations of patients with advanced liver cirrhosis (child B and C, $n=106$ ) were excluded (Fig. 1). All other ablations were included in the study.

Tumor diagnosis was made using typical imaging appearances on multiphasic contrast MRI or CT in addition to histopathological confirmation before or during the SRFA procedure where imaging was inconclusive.

\section{Multi-probe stereotactic radiofrequency ablation}

The basic principle of multi-probe RF ablation with intraoperative image fusion is to produce multiple overlapping ablation zones to entirely cover tumors with a sufficient safety margin (Fig. 2), allowing for larger tumor ablations than single ablation zones. Stereotactic technique relates the position of targets and entry points to a Cartesian coordinate system, and therefore allows for reliable planning and execution of interventional procedures, in this case tumor ablation.

\section{Stereotactic radiofrequency ablation procedure}

The method of SRFA with image fusion for planning, intraoperative verification of coaxial needle placement, and ablation result has been previously described in detail [15]. In brief, the key steps are as follows:

I. Preparation: treatment is performed under general anesthesia with muscle paralysis and immobilization facilitated by a single (Bluebag, Medical Intelligence Inc.) or double vacuum 


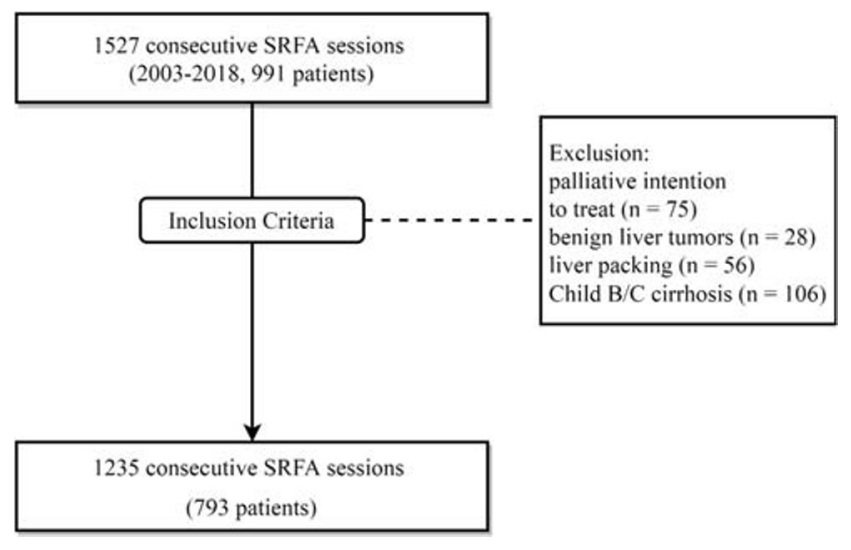

Fig. 1 Flowchart of SRFA selection

fixation technique (BodyFix, Medical Intelligence Inc.); 10-15 broadly attached registration markers (Beekley Spots, Beekley Corporation) are applied to the skin for image-to-patient registration.

II. Planning: a contrast-enhanced CT (Siemens SOMATOM Sensation Open, 82-cm-diameter sliding gantry, Siemens AG) with a 3-mm slice interval is acquired in the axial plane in arterial and venous phases. The obtained CT data is then transferred to an optically based navigation system (Stealth Station Treon plus, Medtronic Inc.) to plan one or multiple trajectories using multiplanar and 3D reconstructed images.

III. Execution - co-axial needle placement: to eliminate respiratory motion, temporary disconnections of the endotracheal tube are performed during the planning CT, during each stereotactic needle placement, and for the final CT. After registration, an accuracy check and sterile draping, the ATLAS aiming device (Elekta PSC Medical Intelligence Inc.), is used for navigated trajectory alignment. $15 \mathrm{G} / 17.2-\mathrm{cm}$ coaxial needles (Bard Inc.) are placed through the aiming device (without real-time imaging) to house radiofrequency electrodes. An unenhanced CT is then performed for verification of correct needle placement and fused with the planning CT using the navigation system's image 3D registration algorithm. A 16G biopsy sample is obtained via one of the coaxial needles for patients without prior histological confirmation.

III. Execution — RF ablation: a maximum of three 17G RFelectrodes (Cool-tip, Medtronic, $25 \mathrm{~cm}$ in length with $3 \mathrm{~cm}$ exposure) are introduced through the coaxial needles for serial tumor ablation. RF ablation is carried out using the unipolar Cool-tip_RF generator (Cool-tip, Medtronic), including the Cool-tip_RF switching controller. The standard ablation time for three electrodes is $16 \mathrm{~min}$ although if a significant increase in impedance (the so-called roll-off effect) is encountered sooner, the ablation process is considered complete. Track ablation is carried out during needle repositioning and final removal to prevent bleeding and potential tumor seeding.

IV. Final control CT: post-ablation contrast-enhanced CT is carried out in the arterial and portal venous phases and fused with the planning $\mathrm{CT}$ to assess ablation zone coverage and complications.

Images from an example SRFA case are shown in Fig. 3.

\section{Adaptations over time}

SRFA was introduced at our institution in 2003 and the following adaptations were made, in chronological order: (i) avoiding pulmonary transgression: 3D planning on multiplanar reconstructed images allows for angulated needle paths, which avoid pleural or pulmonary transgression; (ii) management of
Fig. 2 Illustration of multi-probe RF ablation with multiple overlapping ablation zones (grey ellipsoids with central RF probe) covering the entire tumor volume (yellow sphere); a top view, b oblique view

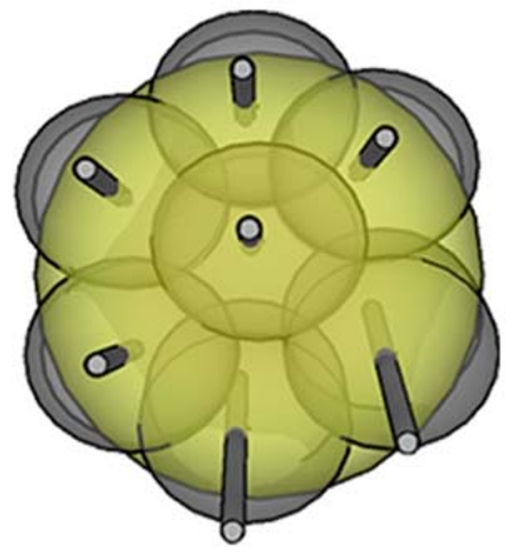

a

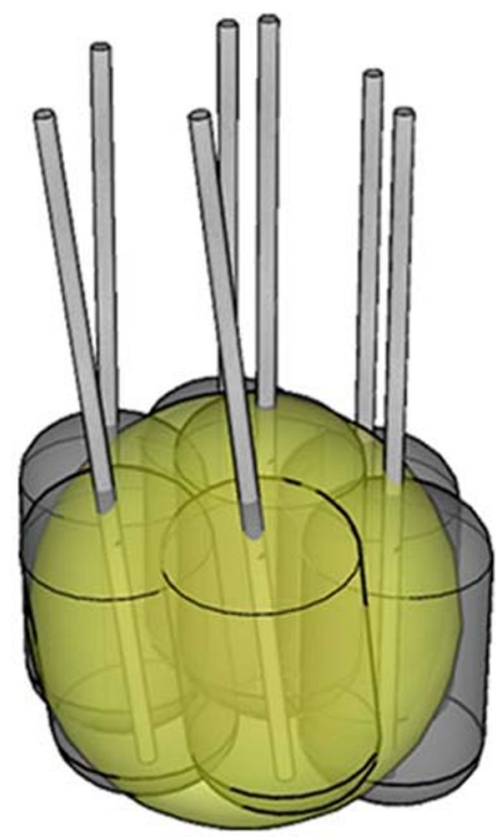

b 
Fig. 3 Case of an 81-year-old male with a single $6.0-\mathrm{cm}$ HCC in segment VII/VIII. a, b Portal venous phase initial CT scan with a hyper-enhancing nodule in segment VII/VIII (red dashed circle). $\mathbf{c}, \mathbf{d}$ Volume rendering and maximum intensity projection (MIP) of the native control CT with 11 needles in place (red arrowhead). e Portal venous phase final control CT scan showing complete ablation zone (red dashed circle). No complications were noted. $\mathbf{f}$ The red dashed circle illustrates the ablation zone at 6 months with no evidence of a late complication or local recurrence
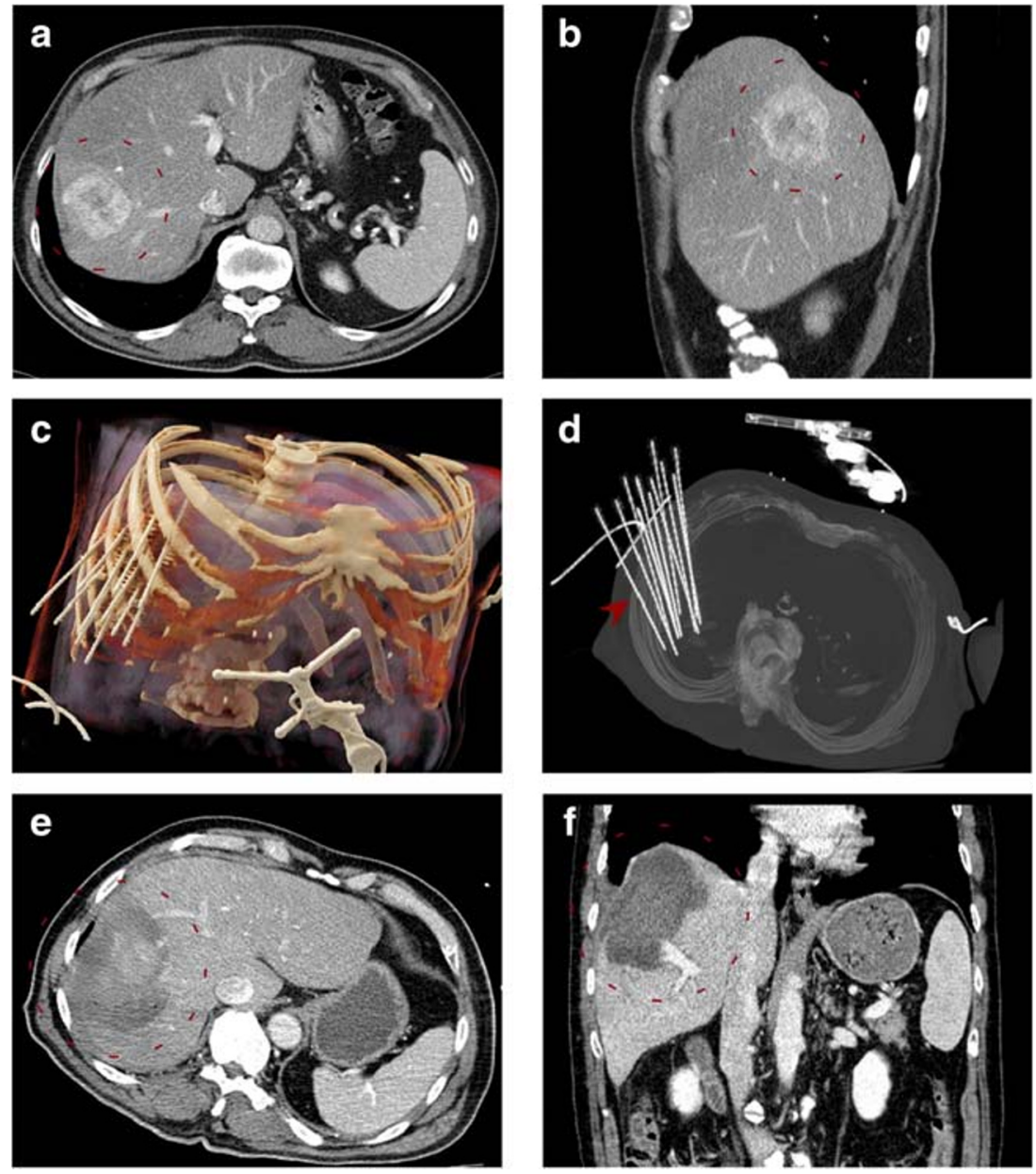

hemorrhage without hemodynamic compromise: 15-min manual external compression was used to treat acute bleeding detected in the final CT; (iii) long-term antibiotics in patients with bilioenteric anastomosis or other risk factors for ascending biliary infection were treated with at least 3 weeks of post-interventional antimicrobial drugs with good biliary penetration; and (iv) management of pleural effusions: pleural effusions were treated with chest drains only after failure of conservative measures.

\section{Complications}

Complications were recorded at the time of SRFA and also evaluated using all available medical records including imaging reports. Major complications were defined according to the Society of Interventional Radiology (SIR) Standards of Practice Committee classification [16]. Perioperative mortality was defined as death occurring within 30 days after ablation.

\section{Definitions of risk factors}

A distance of $<1 \mathrm{~cm}$ was defined as "close to" in terms of the diaphragm, liver capsule, organ, major vessel, central bile duct, or gall bladder. Major vessel was defined as a vessel with a diameter of $>3 \mathrm{~mm}$. Non-critical location was defined as a location without adjacent vulnerable structures. History of bile duct treatment includes sphincteroplasty, papillotomy, and bilioenteric anastomosis.

\section{Statistical analysis}

Statistical analysis was performed using IBM SPSS software version 20. Data were expressed as total numbers, median, and range.

The difference between categorical variables was evaluated with the $X^{2}$ test, whereas the Mann-Whitney $U$ test was used to evaluate independent continuous variables. Binary logistic regression was used to evaluate possible predictors of major complications (categorical outcome) where variables of interest identified in simple logistic regression analysis $(p$ value $<0.1)$ were further analyzed in a multivariable model. A $p$ value $<0.05$ was considered as statistically significant. 


\section{Results}

\section{Patient characteristics (Table 1)}

Seven hundred ninety-three patients, 241 (30.4\%) females and $552(69.6 \%)$ males, with a median age of 65.0 years $(0.3-88.0)$ underwent SRFA for treatment of primary and secondary liver tumors. Diagnoses included $350(44.1 \%)$ hepatocellular carcinomas (HCCs), $36(4.5 \%)$ intrahepatic cholangiocarcinomas (ICCs), and $407(51.3 \%)$ metastatic tumors, whereby the majority (218) were of colorectal origin. The median size of the 2475 nodules was $3.0 \mathrm{~cm}(0.5-19.0 \mathrm{~cm})$. Patients had a median of 2 tumors (1-29) treated in 1235 sessions. At the beginning of treatment, $433(54.7 \%)$ patients had a solitary tumor in the liver, $186(23.5 \%)$ had two tumors, $83(10.5 \%)$ had three tumors, and 91 $(11.3 \%)$ had more than three tumors (defined as multiple tumors). Two hundred ninety-four $(37.1 \%)$ patients had underlying cirrhosis.

\section{Perioperative mortality (Table 2)}

Thirty-day mortality was $0.5 \%(6 / 1235)$ with four deaths occurring from major hemorrhage, one due to sepsis, and one as a consequence of acute-on-chronic renal failure.

\section{Major complications}

Major perioperative complications are shown in Table 3, where the major complication rate was $7.4 \%$ (91 of 1235 ablation sessions). In addition to the aforementioned fatal complications, in descending order of severity, transient liver failure (1 patient), transient respiratory failure (5 patients), and transient cardiac problems (3 patients) required intensive care unit admission (Fig. 4). Thermal damage to hollow viscera ( 3 patients) and the skin ( 2 patients) had to be surgically repaired. Thermal injury to central bile ducts required ERCP and US-guided drainage. One case of thermal injury along the needle track resulted in a pleurocutaneous fistula which required repeated US-guided drainages. Eleven liver abscesses, with bilio-pleural fistulation in two cases, required surgical (6 patients) or interventional (US-guided drainages, 5 patients) treatment. $54.5 \%$ of the patients with abscesses had a positive history of bile duct surgery or intervention. One arterio-portal fistula was treated with angiographic coiling. Other complications included peri-/intrahepatic hemorrhage (30 patients) requiring angiographic coiling, major pleural effusions (11 patients) requiring thoracocentesis, and pneumothorax (16 patients) requiring chest tube insertion.
Table 1 Patient characteristics of 793 patients undergoing 1235 SRFA sessions for 2475 tumors

Patient characteristics

\begin{tabular}{lc}
\hline Age, years (range) & $65.0(0.3-88.0)$ \\
Sex (female/male), $n(\%)$ & $241 / 552(30.4 / 69.6)$ \\
Tumor type, $n(\%)$ & \\
HCC, $n(\%)$ & $350(44.1)$ \\
ICC, $n(\%)$ & $36(4.5)$ \\
Metastasis, $n(\%)$ & $407(51.3)$ \\
Colorectal, $n(\%)$ & $218(61.2)$ \\
Other, $n(\%)$ & $189(38.8)$ \\
Cirrhosis, $n(\%)$ & $294(37.1)$ \\
Number of tumors at initial SRFA & \\
$n=1, n(\%)$ & $433(54.7)$ \\
$n=2, n(\%)$ & $186(23.5)$ \\
$n=3, n(\%)$ & $83(10.5)$ \\
$n>3, n(\%)$ & $91(11.3)$ \\
Tumor size at initial SRFA & \\
$<3$ cm, $n(\%)$ & $382(48.0)$ \\
$3-5$ cm, $n(\%)$ & $275(34.8)$ \\
$>5$ cm, $n(\%)$ & $136(17.2)$ \\
At initial SRFA & \\
Tumors, median (range) & $1.9(0.5-19.0)$ \\
Tumor size, median (range) & $1(1-10)$ \\
Overall treated & $1(1-9)$ \\
Tumors, median (range) & \\
Tumor size, median (range) & \\
Median no. of ablations per patient & \\
& \\
&
\end{tabular}

SRFA stereotactic radiofrequency ablation, HCC hepatocellular carcinoma, ICC intrahepatic cholangiocarcinoma

\section{$50.5 \%(46 / 91)$ of all major complications were successfully treated in the same anesthetic session by angiographic coiling for hemorrhage and chest tube insertion for pneumothorax.}

\section{Risk factors for major complications}

Simple logistic regression analysis showed that significant predictors of major complications were (i) history of bile duct surgery or intervention $(p=0.021)$, (ii) number of coaxial needles (for RF probe positioning) $(p<0.001)$, (iii) number of ablated tumors $(p=0.030$ ), (iv) total tumor size per ablation session $(p<0.001)$, (v) location close to the diaphragm $(p=0.010)$, (vi) tumor conglomerate $(p=0.022)$, and (vii) location in segment IVa $(p=0.021)$, (viii) segment VII $(p=0.018)$, and (ix) segment VIII $(p<0.001)$. After multivariable analysis, history of bile duct surgery/intervention $(p=0.013, \mathrm{OR}=3.290)$, number of coaxial needles $(p=0.026$, OR $=1.052)$, and location of 
Table 2 Details of perioperative deaths

\begin{tabular}{|c|c|c|c|c|c|c|c|c|c|}
\hline ID & Age & Sex & Prim. & Cirr. & $\begin{array}{l}\mathrm{T} / \\
\mathrm{S}\end{array}$ & $\mathrm{mS} / \mathrm{S}$ & $\begin{array}{l}\mathrm{N} / \\
\mathrm{S}\end{array}$ & Fatal complication & Therapy \\
\hline 1 & 77 & Male & $\mathrm{CRC}$ & - & 3 & $5.5 \mathrm{~cm}$ & 12 & Major hemorrhage, hemorrhagic shock (0 days) & AG-coiling, ICU \\
\hline 2 & 83 & Female & $\mathrm{HCC}$ & - & 3 & $4.8 \mathrm{~cm}$ & 12 & Major hemorrhage, MODS (19 days) & AG-coiling, ICU \\
\hline 3 & 73 & Male & $\mathrm{HCC}$ & Child A & 1 & $5.5 \mathrm{~cm}$ & 3 & Major hemorrhage, MODS (20 days) & AG-coiling, ICU \\
\hline 4 & 75 & Male & ICC & - & 1 & $8.0 \mathrm{~cm}$ & 6 & Major hemorrhage, MODS (18 days) & AG-coiling, ICU \\
\hline 5 & 79 & Male & $\mathrm{CRC}$ & - & 1 & $6.5 \mathrm{~cm}$ & 9 & Liver abscess, septic shock (21 days) & Surgery, ICU \\
\hline 6 & 58 & Male & $\mathrm{HCC}$ & Child A & 3 & $1.5 \mathrm{~cm}$ & 4 & Acute on chronic renal failure ( 24 days) & ICU \\
\hline
\end{tabular}

$N / S$ needles per session, $H C C$ hepatocellular carcinoma, $I C C$ intrahepatic cholangiocarcinoma, $C R C$ colorectal carcinoma, $C i r r$. cirrhosis, $A G$ angiography, US ultrasound

tumors in segment IVa $(p=0.016 .013, \mathrm{OR}=1.989)$ or VIII $(p=$ $0.038, \mathrm{OR}=1.635)$ were found to be independent prognostic factors (Table 4, Fig. 4).

The median summated size of treated tumors and number of coaxial needles (for RF probe positioning) was $6.2 \mathrm{~cm}(0.5-$ 23 ) and 9 (1-33), respectively, in sessions associated with complications vs. $3.7 \mathrm{~cm}(0.5-31)$ and $6(1-38)$ for those without complications whereby differences reached statistical significance ( $p<0.001$ for both comparisons). The median ablation time per session was $32 \mathrm{~min}$ (10-330 $\mathrm{min}$ ).

With regard to liver abscesses as one of the most serious complications, a positive history of bile duct surgery or interventions showed a significant impact on their occurrence: Liver abscesses occurred significantly more frequently in patients with bile duct history with $18.2 \%$ (6/33) vs. $0.5 \%$ $(6 / 1202)(p=0.000)$.

Fever ( $>37^{\circ} \mathrm{C}$, measured peripherally) developed in all patients but subsided in 1-2 days with symptomatic treatment.
Median postoperative hospital stay was 4 days (1-42) and significantly longer in patients who developed major complications with median 7.5 days $(3-42)(p<0.001)$.

\section{Adaptations over time (Fig. 5)}

Several adaptations, such as sparing of the pleural recess, external compression in case of post-procedural hemorrhage without hemodynamic compromise, more conservative management of pleural effusions, and long-term antibiotic treatment in case of pneumobilia or bile duct surgery, lead to an overall decrease of the major complication rate from $11.5 \%$ before January 2011 to $6.0 \%(36 / 314,55 / 921, p=0.001)$. Rates of the most common complications, namely pneumothorax, major hemorrhage, pleural effusion, and liver abscesses, decreased from 2.2 to $1 \%$ (7/314, 9/921, $p=0.09), 4.1$ to $1.8 \%(13 / 314,17 / 921, p=$ $0.023), 1.9$ to $0.5 \%(6 / 314,5 / 921, p=0.026)$, and 1.9 to $0.7 \%$ $(6 / 314,6 / 921, p=0.049)$, respectively.

Table 3 Major complications after SRFA

\begin{tabular}{llll}
\hline Complication & No. & Therapy & Treated in same GA session \\
\hline Hemorrhagic shock & 4 & AG-coiling, ICU, death (4) & - \\
Septic shock, liver abscess (1) & 1 & ICU, surgery, drainage, death (1) & - \\
Acute on chronic renal failure & 1 & ICU, death (1) & - \\
Transient liver failure & 1 & ICU & - \\
Transient respiratory failure & 5 & ICU & - \\
Transient cardiac problems & 3 & ICU & - \\
Thermal injury of hollow visceral organs & 3 & Surgery & - \\
Thermal injury of the skin & 2 & Surgery & - \\
Thermal injury of the bile duct & 1 & ERCP & - \\
Thermal injury of the pleura and skin with fistulation & 1 & repeated US drainages & - \\
Liver abscess, w. bilio-pleural fistula (2) & 11 & Surgery (6), US-guided drainage (5) & - \\
Pleural effusion & 11 & Thoracentesis & 17 \\
Arterio-portal fistula & 1 & AG-coiling & 34 \\
Pneumothorax & 16 & Thoracostomy tube & AG-Coiling \\
Peri-/intrahepatic hemorrhage & 30 & . & \\
\hline
\end{tabular}

SRFA stereotactic radiofrequency ablation, No. number, ICU intensive care unit, $A G$ angiography, $U S$ ultrasound, GA general anesthesia 


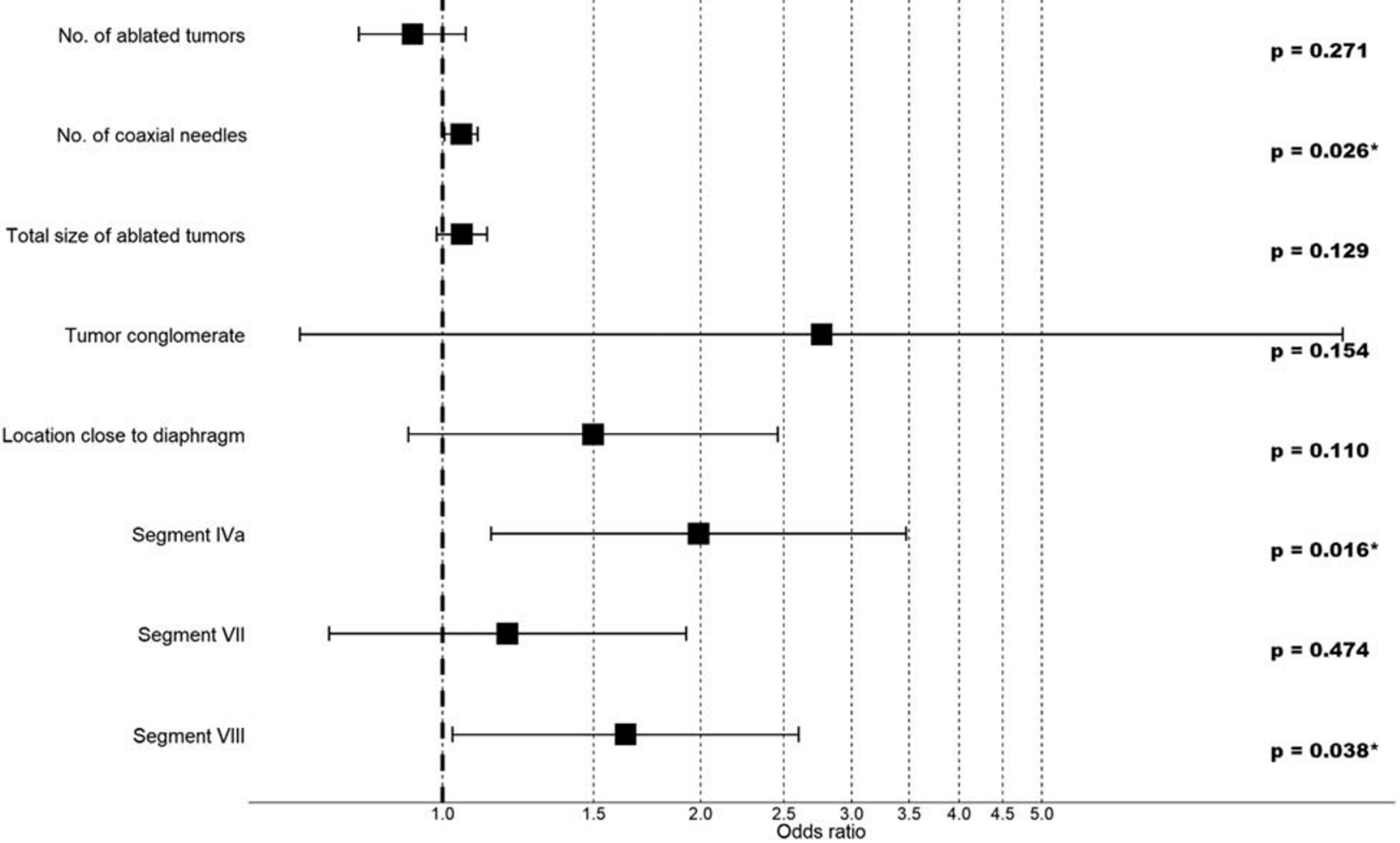

Fig. 4 Forest plot of multivariable logistic regression analysis of major complications

\section{Efficacy and overall survival}

The overall complete ablation rate in the present study was $96.2 \%$ (2382/2475 tumors). Based on tumor type, the complete ablation rate was $96.2 \%$ (957/995) for HCC, $94.5 \%$ (137/145) for ICC, and 95.5\% (1288/1335) for liver metastasis. The corresponding overall local recurrence rate was $8.3 \%$ (205/2475) and 7.2\% (72/995) for HCC, $13.8 \%$ (20/145) for ICC, and $8.5 \%$ (113/1335) for liver metastasis.

The overall survival (OS) rate for the entire study cohort was $89 \%, 60 \%$, and $44 \%$ at 1 year, 3 years, and 5 years, respectively, with a median OS of 50 months (95 CI 43.956.4). Based on tumor type, the OS rates at 1 year, 3 years, and 5 years were $93 \%, 69 \%$, and $53 \%$ for $\mathrm{HCC} ; 82 \%, 51 \%$, and $43 \%$ for ICC; and $86 \%, 53 \%$, and $36 \%$ for liver metastasis.

\section{Discussion}

In this series of 1235 ablation sessions over 15 years, we have shown that stereotactic radiofrequency ablation (SRFA) provides low levels of morbidity and mortality in the treatment of primary and metastatic liver tumors with a mortality of $0.5 \%$ (6/1235) and an overall major complication rate of 7.4\% (91/
1235). Furthermore, we found the major complication rate decreased from 11.5 (36/314) before January 2011 to $6.0 \%$ (55/921) after January 2011 which could be attributable to procedural adaptations, increasing experience, and improved patient selection.

Major complication rates in the present study are similar to those quoted in the conventional tumor ablation literature (i.e., without navigation systems) of $0.9-10.0 \%[4,8,9]$, despite our selection of large $(17 \%>5 \mathrm{~cm})$ and multiple tumors $(21.8 \% n \geq 3)$. This observation could reflect the additional safety that is offered by stereotactic guidance through careful planning and precision targeting.

The only independent predictive factors for major complications were history of bile duct surgery/intervention, number of coaxial needles, and location of tumors in segment IVa or VIII. Total tumor size was shown to be predictive of major complications at simple logistic regression analysis, although it was not an independent predictive factor at multivariable analysis.

In the present study, we have excluded patients with deteriorated liver function (child $\mathrm{B}$ and $\mathrm{C}$ cirrhosis) because the associated major complication of liver failure after treatment cannot be avoided by the adaptation or improvement of treatment technique. This is in contrast to technique-related 
Table 4 Uni- and multivariable logistic regression analyses of risk factors for major complications after a learning curve

\begin{tabular}{|c|c|c|c|c|c|c|}
\hline \multirow[t]{2}{*}{ Variables } & \multirow[t]{2}{*}{ No. of sessions } & \multirow[t]{2}{*}{ Major compl. (\%) } & \multirow[t]{2}{*}{ Simple A. $p$ value } & \multicolumn{3}{|c|}{ Multivariable analysis } \\
\hline & & & & $p$ value & Odds ratio & $95 \% \mathrm{CI}$ \\
\hline Age $(>70 /<70)$ & $386 / 849$ & $8.5 / 6.8$ & 0.29 & - & - & - \\
\hline Sex (female/male) & $350 / 885$ & $8.3 / 7.0$ & 0.44 & - & - & - \\
\hline Tumor type (HCC/ICC/metastatic) & $538 / 86 / 611$ & $6.1 / 7.0 / 8.5$ & 0.13 & - & - & - \\
\hline Liver cirrhosis $(+/-)$ & $441 / 794$ & $5.9 / 8.2$ & 0.14 & - & - & - \\
\hline No. of needles & 1235 & - & $<0.001 *$ & $0.026^{*}$ & 1.052 & $1.006-1.099$ \\
\hline No. of ablated tumors & 1235 & - & $0.030 *$ & 0.271 & 0.923 & $0.799-1.065$ \\
\hline Total size of ablated tumors & 1235 & - & $<0.001 *$ & 0.129 & 1.054 & $0.985-1.127$ \\
\hline $\mathrm{BD}$ treatment ${ }^{ \pm}$ & $33 / 1202$ & $18.2 / 7.1$ & $0.021 *$ & $0.013 *$ & 3.290 & $1.285-8.421$ \\
\hline \multicolumn{7}{|l|}{ Location close to } \\
\hline Diaphragm & $250 / 985$ & $11.2 / 6.4$ & $0.010 *$ & 0.110 & 1.499 & $0.913-2.461$ \\
\hline Liver capsule & $581 / 654$ & $8.6 / 6.3$ & 0.12 & - & - & - \\
\hline Organ & $188 / 1047$ & $7.4 / 7.4$ & 0.96 & - & - & - \\
\hline Major vessel & $361 / 874$ & $8.6 / 6.9$ & 0.52 & - & - & - \\
\hline Central bile duct & $41 / 1194$ & $12.2 / 7.2$ & 0.24 & - & - & - \\
\hline Gall bladder & $46 / 1189$ & $8.7 / 7.3$ & 0.73 & - & - & - \\
\hline Non-critical location & $454 / 781$ & 7.0/7.6 & 0.74 & - & - & - \\
\hline Conglomerate & $11 / 1224$ & $27.3 / 7.2$ & $0.022 *$ & 0.154 & 2.767 & $0.682-11.224$ \\
\hline Liver segments & & & & - & - & - \\
\hline Segment I & $81 / 1154$ & $11.1 / 7.1$ & 0.19 & - & - & - \\
\hline Segment II & $224 / 1011$ & $4.9 / 7.9$ & 0.12 & - & - & - \\
\hline Segment III & $198 / 1037$ & $8.1 / 7.2$ & 0.68 & - & - & - \\
\hline Segment IVa & $160 / 1075$ & $11.9 / 6.7$ & $0.021 *$ & $0.016^{*}$ & 1.989 & $1.139-3.470$ \\
\hline Segment IVb & $93 / 1142$ & $7.5 / 7.4$ & 0.95 & - & - & - \\
\hline Segment V & $266 / 969$ & $6.0 / 7.7$ & 0.34 & - & - & - \\
\hline Segment VI & $355 / 880$ & $8.5 / 6.9$ & 0.36 & - & - & - \\
\hline Segment VII & $466 / 769$ & $9.7 / 6.0$ & $0.018^{*}$ & 0.474 & 1.191 & $0.738-1.924$ \\
\hline Segment VIII & $504 / 731$ & $10.5 / 5.2$ & $<0.001 *$ & $0.038^{*}$ & 1.635 & $1.028-2.601$ \\
\hline
\end{tabular}

HCC hepatocellular carcinoma, ICC intrahepatic cholangiocarcinoma, No. number, Compl. complication

${ }^{ \pm}$Includes sphincteroplasty, papillotomy

* Statistically significant

complications such as hemorrhage, pleural effusion, or pneumothorax; their occurrence can be reduced or even prevented by technical improvements of SRFA compared to conventional RFA.

The historic literature suggests that percutaneous RFA of liver tumors in difficult locations leads to more complications, insufficient ablation, and poor tumor control [17, 18], particularly in the case of subcapsular lesions $[14,17,19,20]$. However, more recently, studies have reported similar complication rates for subcardiac vs. non-subcardiac tumors (1-7.7\%) [21-23]. In line with these reports, we found that subcapsular tumors were not associated with higher major complication rates following logistic regression analysis $(p=0.12)$. Lesions high in the liver require either transthoracic $[20,24,25]$ or steeply angulated transhepatic approaches [26], with some investigators using adjunctive hydrodissection techniques [27]. Lower major complication rates of $10 \%(6 / 60)$ have been reported using transhepatic approaches [28] vs. 57.9\% for transpleural approaches [29], which is why we favor the former. However, angulated multiprobe ablations pose severe technical challenges which in practice limits the size of lesions that can be treated without stereotactic guidance. While laparoscopic RFA can also be used to treat difficult lesions, it is more invasive with higher rates of reported major complications at $10-12.4 \%[28,30]$. Surgical resection is associated with even higher major complication rates at $10-27 \%$ for laparoscopic and 18-37\% for open resection [31-35]. Simple logistic regression analysis showed that lesions close to the diaphragm at the hepatic dome were a predictor of major complication $(11.2 \%$ vs. $6.4 \%)$; however, this was not found to be an independent risk factor at multivariable analysis. 

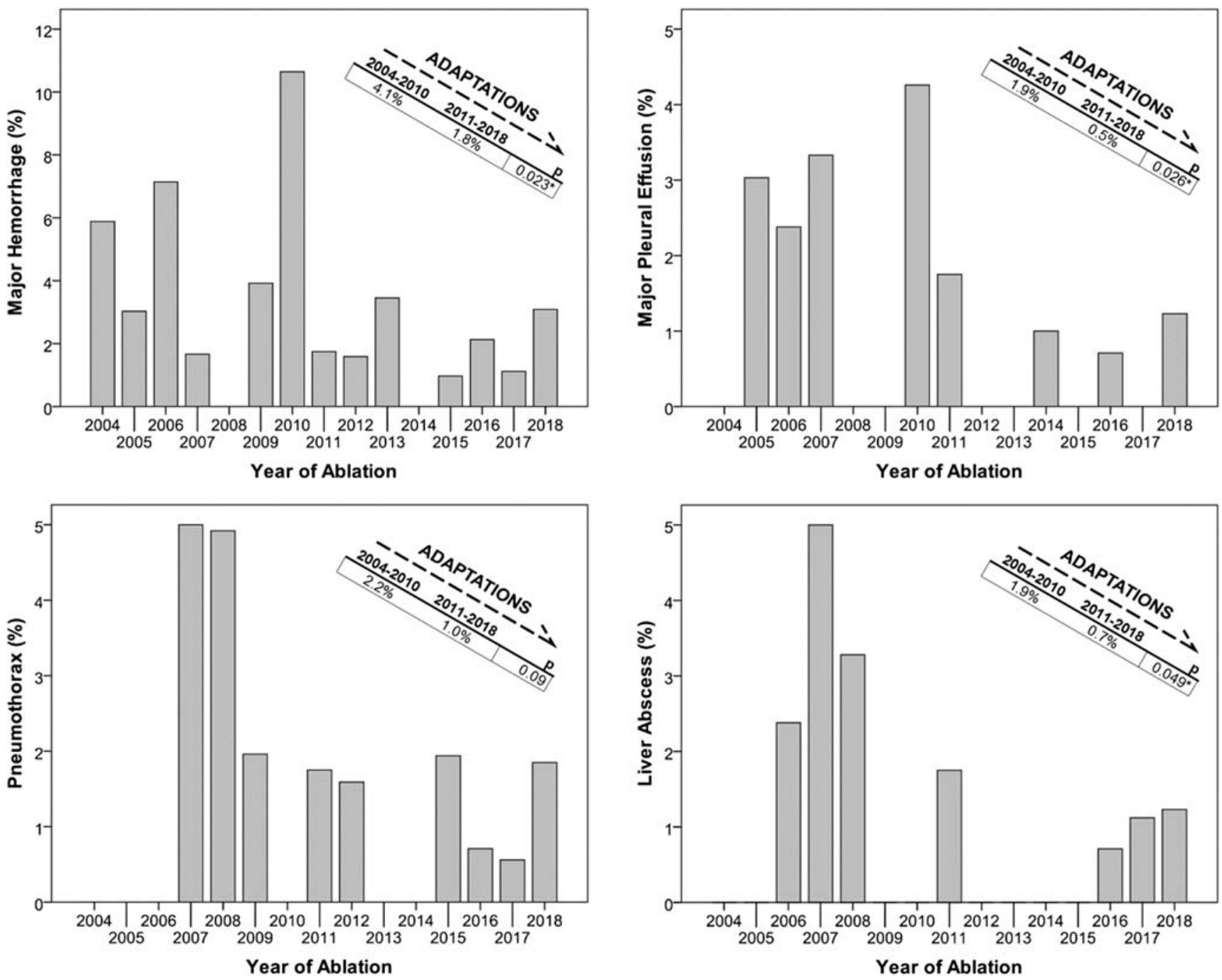

Fig. 5 Overview of the most frequent major complications between 2004 and 2018

Furthermore, $62.6 \%$ of major complications including pleural effusion, pneumothorax, and major hemorrhage could be easily managed with a single interventional procedure and $50.5 \%$ were managed in the same general anesthetic session without changing the postoperative course.

Several adaptations resulted in a significant decrease in major complication rate from $11.5 \%$ before January 2011 to $6.0 \%(p=0.001)$. Specifically, sparing of the pleural recess resulted in a decrease in pneumothorax from 2.2 to $1.0 \%$ ( $p=$ 0.09). However, the risk for pneumothorax remains, since lesions located in the liver dome may require paths through the pleural recess. Post-procedural (usually perihepatic) hemorrhage without hemodynamic compromise was managed with manual external compression, which reduced the rate of subsequent angiographic coiling from 4.1 to $1.8 \%(p=0.023)$. A more conservative, non-inferior, management of pleural effusions leads to a decrease in thoracentesis from 1.9 to $0.5 \%(p=0.026)$. In case of pneumobilia or bile duct surgery, long-time antibiotic treatment resulted in a decreased rate of liver abscesses from 1.9 to $0.7 \%(p=0.049)$.

The limitations of our study include its retrospective design and single treatment center bias, which reduces the generalizability of our data. Limited use of navigation systems as part of ablation procedures also reduces the generalizability, although use is gaining popularity.

In conclusion, stereotactic radiofrequency ablation (SRFA) of liver tumors is safe and can extend the treatment spectrum of conventional RFA, whereby history of bile duct surgery/ intervention, number of coaxial needles, and location of tumors in segment IVa or VIII are the statistically most important risk factors for major complications. Adaptations over time with increasing experience lead to a significant decrease in complications.

Funding Open access funding provided by University of Innsbruck and Medical University of Innsbruck. 


\section{Compliance with ethical standards}

Guarantor The scientific guarantor of this publication is Reto Bale.

Conflict of interest The authors of this manuscript declare no relationships with any companies whose products or services may be related to the subject matter of the article.

Statistics and biometry One of the authors (PS) has significant statistical expertise.

Informed consent Written informed consent was obtained from all subjects (patients) in this study.

Ethical approval Institutional Review Board approval was obtained.

Study subjects or cohorts overlap Of the 883 patients, 545 have been previously reported:

1Schullian P, Putzer D, Laimer G, Levy E, Bale R (2020) Feasibility, safety, and long-term efficacy of stereotactic radiofrequency ablation for tumors adjacent to the diaphragm in the hepatic dome: a case-control study. Eur Radiol 30:950-960

2Schullian P, Johnston EW, Putzer D, Eberle G, Laimer G, Bale R (2020) Safety and efficacy of stereotactic radiofrequency ablation for very large $(>/=8 \mathrm{~cm})$ primary and metastatic liver tumors. Sci Rep 10:1618

3Schullian P, Putzer D, Silva MA, Laimer G, Kolbitsch C, Bale R (2019) Stereotactic radiofrequency ablation of liver tumors in octogenarians. Front Oncol 9:929

4Schullian P, Laimer G, Putzer D, Effenberger M, Bale R (2019) Stereotactic radiofrequency ablation of primary liver tumors in the caudate lobe. HPB (Oxford). 10.1016/j.hpb.2019.09.008

5Schullian P, Johnston EW, Putzer D, Eberle G, Laimer G, Bale R (2019) Stereotactic radiofrequency ablation of subcardiac hepatocellular carcinoma: a case-control study. Int J Hyperthermia 36:876-885

6Bale R, Richter M, Dunser M, Levy E, Buchberger W, Schullian P (2018) Stereotactic radiofrequency ablation for breast cancer liver metastases. J Vasc Interv Radiol 29:262-267

7Bale R, Schullian P, Schmuth M, Widmann G, Jaschke W, Weinlich G (2016) Stereotactic radiofrequency ablation for metastatic melanoma to the liver. Cardiovasc Intervent Radiol 39:1128-1135

8Widmann G, Schullian P, Haidu M, Bale R (2012) Stereotactic radiofrequency ablation (SRFA) of liver lesions: technique effectiveness, safety, and interoperator performance. Cardiovasc Intervent Radiol 35:570-580

9Bale R, Widmann G, Schullian P et al (2012) Percutaneous stereotactic radiofrequency ablation of colorectal liver metastases. Eur Radiol 22:930-937

These prior articles dealt with complications only as a part objective whereas the current study expands on this by having a much larger patient number and includes new and much more profound analysis and comparisons.

\section{Methodology \\ - retrospective \\ - observational \\ - performed at one institution}

Open Access This article is licensed under a Creative Commons Attribution 4.0 International License, which permits use, sharing, adaptation, distribution and reproduction in any medium or format, as long as you give appropriate credit to the original author(s) and the source, provide a link to the Creative Commons licence, and indicate if changes were made. The images or other third party material in this article are included in the article's Creative Commons licence, unless indicated otherwise in a credit line to the material. If material is not included in the article's Creative Commons licence and your intended use is not permitted by statutory regulation or exceeds the permitted use, you will need to obtain permission directly from the copyright holder. To view a copy of this licence, visit http://creativecommons.org/licenses/by/4.0/.

\section{References}

1. Dokmak S, Fteriche FS, Borscheid R, Cauchy F, Farges O, Belghiti J (2013) 2012 Liver resections in the 21st century: we are far from zero mortality. HPB (Oxford) 15:908-915

2. Reuter NP, Woodall CE, Scoggins CR, McMasters KM, Martin RC (2009) Radiofrequency ablation vs. resection for hepatic colorectal metastasis: therapeutically equivalent? J Gastrointest Surg 13:486491

3. Egger ME, Ohlendorf JM, Scoggins CR, McMasters KM, Martin RC 2nd (2015) Assessment of the reporting of quality and outcome measures in hepatic resections: a call for 90-day reporting in all hepatectomy series. HPB (Oxford) 17:839-845

4. Livraghi T, Meloni F, Di Stasi M et al (2008) Sustained complete response and complications rates after radiofrequency ablation of very early hepatocellular carcinoma in cirrhosis: Is resection still the treatment of choice? Hepatology 47:82-89

5. Livraghi T, Solbiati L (2001) Percutaneous treatment: radiofrequency ablation of hepatic metastases in colorectal cancer. Tumori 87:S69

6. Taner T, Atwell TD, Zhang L et al (2013) Adjunctive radiofrequency ablation of metastatic neuroendocrine cancer to the liver complements surgical resection. HPB (Oxford) 15:190-195

7. Wang JH, Wang CC, Hung CH, Chen CL, Lu SN (2012) Survival comparison between surgical resection and radiofrequency ablation for patients in BCLC very early/early stage hepatocellular carcinoma. J Hepatol 56:412-418

8. Kong WT, Zhang WW, Qiu YD et al (2009) Major complications after radiofrequency ablation for liver tumors: analysis of 255 patients. World J Gastroenterol 15:2651-2656

9. Livraghi T, Solbiati L, Meloni MF, Gazelle GS, Halpern EF, Goldberg SN (2003) Treatment of focal liver tumors with percutaneous radio-frequency ablation: complications encountered in a multicenter study. Radiology 226:441-451

10. Shady W, Petre EN, Gonen M et al (2016) Percutaneous radiofrequency ablation of colorectal cancer liver metastases: factors affecting outcomes-a 10-year experience at a single center. Radiology 278:601-611

11. Liao M, Zhong X, Zhang J et al (2017) Radiofrequency ablation using a $10-\mathrm{mm}$ target margin for small hepatocellular carcinoma in patients with liver cirrhosis: A prospective randomized trial. J Surg Oncol 115:971-979

12. Laimer G, Schullian P, Jaschke N et al (2020) Minimal ablative margin (MAM) assessment with image fusion: an independent predictor for local tumor progression in hepatocellular carcinoma after stereotactic radiofrequency ablation. Eur Radiol. https://doi.org/10. 1007/s00330-019-06609-7

13. Shirai K, Tamai H, Shingaki $N$ et al (2011) Clinical features and risk factors of extrahepatic seeding after percutaneous radiofrequency ablation for hepatocellular carcinoma. Hepatol Res 41:738-745

14. Teratani T, Yoshida H, Shiina S et al (2006) Radiofrequency ablation for hepatocellular carcinoma in so-called high-risk locations. Hepatology 43:1101-1108

15. Bale R, Widmann G, Schullian P et al (2012) Percutaneous stereotactic radiofrequency ablation of colorectal liver metastases. Eur Radiol 22:930-937 
16. Omary RA, Bettmann MA, Cardella JF et al (2003) Quality improvement guidelines for the reporting and archiving of interventional radiology procedures. J Vasc Interv Radiol 14:S293-S295

17. Chen MH, Yang W, Yan K et al (2008) Radiofrequency ablation of problematically located hepatocellular carcinoma: tailored approach. Abdom Imaging 33:428-436

18. Feng K, Yan J, Li X et al (2012) A randomized controlled trial of radiofrequency ablation and surgical resection in the treatment of small hepatocellular carcinoma. J Hepatol 57:794-802

19. Komorizono Y, Oketani M, Sako K et al (2003) Risk factors for local recurrence of small hepatocellular carcinoma tumors after a single session, single application of percutaneous radiofrequency ablation. Cancer 97:1253-1262

20. Shibata T, Maetani Y, Kubo T, Itoh K, Togashi K, Hiraoka M (2004) Transthoracic percutaneous radiofrequency ablation for liver tumors in the hepatic dome. J Vasc Interv Radiol 15:1323-1327

21. Cha DI, Kang TW, Song KD et al (2018) Radiofrequency ablation for subcardiac hepatocellular carcinoma: therapeutic outcomes and risk factors for technical failure. Eur Radiol. https://doi.org/10. 1007/s00330-018-5868-2

22. Kim YS, Lee WJ, Rhim H, Lim HK, Choi D, Lee JY (2010) The minimal ablative margin of radiofrequency ablation of hepatocellular carcinoma ( $>2$ and $<5 \mathrm{~cm}$ ) needed to prevent local tumor progression: 3D quantitative assessment using CT image fusion. AJR Am J Roentgenol 195:758-765

23. Schullian P, Johnston EW, Putzer D, Eberle G, Laimer G, Bale R (2019) Stereotactic radiofrequency ablation of subcardiac hepatocellular carcinoma: a case-control study. Int J Hyperthermia 36: 876-885

24. Park BJ, Byun JH, Jin YH et al (2009) CT-guided radiofrequency ablation for hepatocellular carcinomas that were undetectable at US: therapeutic effectiveness and safety. J Vasc Interv Radiol 20: 490-499

25. Toyoda M, Kakizaki S, Horiuchi K et al (2006) Computed tomography-guided transpulmonary radiofrequency ablation for hepatocellular carcinoma located in hepatic dome. World $\mathrm{J}$ Gastroenterol 12:608-611
26. Kim YK, Kim CS, Lee JM, Chung GH, Chon SB (2006) Efficacy and safety of radiofrequency ablation of hepatocellular carcinoma in the hepatic dome with the CT-guided extrathoracic transhepatic approach. Eur J Radiol 60:100-107

27. Song I, Rhim H, Lim HK, Kim YS, Choi D (2009) Percutaneous radiofrequency ablation of hepatocellular carcinoma abutting the diaphragm and gastrointestinal tracts with the use of artificial ascites: safety and technical efficacy in 143 patients. Eur Radiol 19: 2630-2640

28. Ding H, Su M, Zhu C, Wang L, Zheng Q, Wan Y (2017) CTguided versus laparoscopic radiofrequency ablation in recurrent small hepatocellular carcinoma against the diaphragmatic dome. Sci Rep 7:44583

29. Vo Chieu VD, Werncke T, Hensen B, Wacker F, Ringe KI (2018) CT-guided microwave ablation of liver tumors in anatomically challenging locations. Cardiovasc Intervent Radiol 41:1520-1529

30. Mulier S, Mulier P, Ni Y et al (2002) Complications of radiofrequency coagulation of liver tumours. Br J Surg 89:1206-1222

31. Cho JY, Han HS, Yoon YS, Shin SH (2008) Feasibility of laparoscopic liver resection for tumors located in the posterosuperior segments of the liver, with a special reference to overcoming current limitations on tumor location. Surgery 144:32-38

32. Kaneko H, Takagi S, Otsuka Y et al (2005) Laparoscopic liver resection of hepatocellular carcinoma. Am J Surg 189:190-194

33. D'Hondt M, Tamby E, Boscart I et al (2018) Laparoscopic versus open parenchymal preserving liver resections in the posterosuperior segments: a case-matched study. Surg Endosc 32:1478-1485

34. Castaing D, Vibert E, Ricca L, Azoulay D, Adam R, Gayet B (2009) Oncologic results of laparoscopic versus open hepatectomy for colorectal liver metastases in two specialized centers. Ann Surg 250:849-855

35. Xiao L, Xiang LJ, Li JW, Chen J, Fan YD, Zheng SG (2015) Laparoscopic versus open liver resection for hepatocellular carcinoma in posterosuperior segments. Surg Endosc 29:2994-3001

Publisher's note Springer Nature remains neutral with regard to jurisdictional claims in published maps and institutional affiliations. 